KCL-PH-TH/2010-20

\title{
Stringy Space-Time Foam, Finsler-like Metrics and Dark Matter Relics
}

\author{
Nick E. Mavromatos, Sarben Sarkar and Ariadne Vergou \\ King's College London, Department of Physics, Strand WC2R 2LS, London, U.K.
}

\begin{abstract}
We discuss modifications of the thermal Dark Matter (DM) relic abundances in stringy cosmologies with D-particle space-time foamy backgrounds. As a result of back-reaction of massive DM on the background space-time, owing to its interaction with D-particle defects in the foam, quantum fluctuations are induced in the space-time metric. We demonstrate that these lead to the presence of extra source terms in the Boltzmann equation used to determine the thermal dark matter relic abundances. The source terms are determined by the specific form of the induced metric deformations; the latter depend on the momentum transfer of the DM particle during its interactions with the D-particle defects and so are akin to Finsler metrics. In the case of low string scales arising from large extra dimensions our results may have phenomenological implications for the search of viable supersymmetric models.
\end{abstract}

\section{INTRODUCTION}

The nature of the Dark sector of our Universe constitutes one of the major unresolved puzzles of modern physics. Indeed, according to observations over the past twelve years, $96 \%$ of our Universe energy budget consists of unknown entities: $23 \%$ is Dark Matter (DM) and $73 \%$ is dark energy (DE), a mysterious form of ground state energy. DE is believed to be responsible for the current-era acceleration of the Universe. These numbers have been obtained by best-fit analyses of a plethora of astrophysical data to the so-called Standard Cosmological Model $(\Lambda$ CDM), which is a Friedman-Robertson-Walker (FRW) cosmology, involving cold DM, as the dominant DM species, and a positive cosmological constant $\Lambda>0$; the data range from direct observations of the Universe acceleration, using type-Ia supernovae [1], to cosmic microwave background [2], baryon oscillation [3] and weak lensing data [4]. It should be stressed that the afore-mentioned energy budget depends crucially on the theoretical model for the Universe considered.

An interesting, and not commonly discussed, class of Cosmological models that may lead to modifications of the Dark sector, involves space-time with a "foamy" structure at microscopic (Planckian or string) scales [5], due to quantum gravitational interactions. In the past, for a variety of reasons, such models (differing in the details of the constructs of space-time foam) have been considered by many authors. They exhibit a profusion of features that can be falsified experimentally and their predictions range from light-cone fluctuations caused by stochastic metric fluctuations [6], to macroscopic Lorentz symmetry violations [7]. In this note we would like to present a first study towards the contribution of such space-time metric stochastic fluctuations on the Dark sector and in particular on the DM sector of the Universe. We shall focus on a particular class of stochastic space-time foam models, inspired by certain types of string theory. These involve localized space-time defects (D0-branes or D-particles) [8] which are either allowed background configurations $[\underline{8}, 9]$ or arise effectively from suitable compactifications of higher dimensional branes (e.g. D3-branes wrapped up in appropriate three cycles in the context of type IIB strings [10]) . Observers on the brane detect a foam-like structure due to the crossing of the brane by D-particles. In this higher-dimensional geometry, only gravitational fields are allowed to propagate in the bulk; all other particle excitations, including DM candidates, are assumed to be described by open strings with their ends attached to the brane world. The brane is assumed to have three large spatial dimensions, and - depending on the model of string theory considered - it may have a number of compactified extra dimensions.

Dynamical D-particles should not be viewed as material excitations of the vacuum but rather as vacuum structures. This contrasts with attempts to represent such D-particles as ordinary superheavy DM excitations from the vacuum [11], owing to the completely localized nature of the D-particles in the extra dimensions. In our construction, closest in spirit to weak coupling string theory, they are just vacuum defects. In fact it can be shown that the gravitational interactions among such (BPS) D-particles are cancelled by appropriate gauged repulsive forces induced on them from other branes in our supersymmetric models of D-foam [8]. Hence such a collection of D-particles in the bulk does not affect the Hubble expansion on the D3-brane worlds, and so their concentration cannot be restricted by considerations on overclosure of the Universe within our models. Important constraints on the density of defects in D-foam models can still be imposed by astrophysical experiments on the arrival times of high energy cosmic photons [12]. According to these string-foam models, there should be an effective refractive index in vacuo, such that higher energy photons would be delayed more, since they would cause stronger disturbance on the background 
space-time [8 10]. The interaction of material open strings (such as photons) with the D-particles leads to an induced distortion of space-time described by a metric, which depends on both the coordinate and momentum transfer of the photon during its scattering with the defect and so has similarities to a Finsler metric [13]. This is a topologically non-trivial process, involving the creation of a non-local intermediate string state, oscillating from zero length to a maximum one, according to a time-space stringy uncertainty [9, 14]. This causes a time delay for the photon emerging after capture by the defect, proportional to the incident energy of the photon. .

The purpose here is to analyze, in the same spirit, the modification of the estimate of the DM budget of the Universe as compared to the $\Lambda \mathrm{CDM}$ model due to the quantum fluctuations of the D-particles. In fact, as we shall argue, the propagation of massive DM particles on such space-times, induces a back-reaction, which in turn has consequences for the amount of thermal DM relics of these particles; this, in turn, impacts on astro-particle tests of particle physics models incorporating supersymmetry, that provide currently one of the leading DM candidate species, the neutralino. Its thermal abundance, calculated within the simplest supersymmetry models (minimal supersymmetric model embedded in minimal supergravity [15]), is heavily restricted by cosmic microwave background data; hence the available parameter space for these simplest supersymmetric models may vanish in the near future, on incorporating also data from collider experiments such as the LHC at CERN [16]. These constraints depend strongly on details of theoretical models. In the presence, for instance, of extended supergravity models with time-dependent dilaton- $\phi$ sources [17], the calculated amount of thermal neutralino relic abundance can be smaller than the one calculated within the $\Lambda$ CDM-minimal supergravity cosmology. Such dilaton models allow more scope for supersymmetry, which can thus survive the otherwise stringent tests at the LHC [18]. In our work we will find that the effects of the D-foam on the thermal relic abundances oppose those from the dilaton models. These effects may become relevant for models allowing low string mass scales.

For clarity we will first outline the basic reason behind such modifications in the DM thermal abundances. Nonequilibrium cosmology models are associated with space-time distortions, due to either the presence of time dependent dilaton sources (cf supercritical (SSC) dilaton quintessence string cosmologies [17]), or the induced back reaction of the DM particles onto the space-time itself. Boltzmann equations are used to determines the thermal DM species cosmic abundances. The effect of space-time distortions can be subsumed as extra contributions to a source $\Gamma(t)$ for particle production (at cosmic Robertson-Walker time $t$ ) on the right-hand side of the appropriate Boltzmann equation. In general (for a Universe with three spatial dimensions (i.e. a 3-brane), with no loss of particles to the bulk ), with $n(t)$ the density of the DM species, the apposite Boltzmann equation reads :

$$
\frac{d n}{d t}+3 H n=\Gamma(t) n+\mathcal{C}[n]
$$

where $H=\frac{\frac{d a}{d t}}{a} \equiv \frac{\dot{a}}{a}$ is the Hubble ratio, $a$ being the scale factor in the Robertson-Walker metric, and $\mathcal{C}[n]$ is the standard collision term describing the deviations of the species population from thermal equilibrium. In standard cosmologies, which we assume here, this has the form [19]

$$
\mathcal{C}[n]=-\langle\tilde{\sigma} v\rangle\left(n^{2}-n_{\text {eq }}^{2}\right)
$$

with $n_{\mathrm{eq}}$ a thermal equilibrium density of species, $\tilde{\sigma}$ the total cross section evaluated in the background metric, and $v$ the Mœller velocity.

We will provide an explicit expression for the D-foam induced source $\Gamma(t)$. The interaction of DM particles, represented as open string excitations on the D3 brane world, with D-particles, (interpreted as space-time defects in the ground-state ), results in metric distortions. The latter depend on the momentum transfer of the matter particles and so are of Finsler type [13]. It was observed that [20], for the universe to expand by a scale factor $a(t)$, in a way consistent with the world-sheet logarithmic conformal invariance of the D-particle recoil process during capture and scattering [21], there is an induced space-time distortion which has the form:

$$
d s^{2}=-d t^{2}+v_{i} a^{2}\left(t_{0}\right) d t d x^{i}+a^{2}(t) d x^{i} d x^{i} \delta_{i j}, \quad t>t_{0} .
$$

Here the underlying space-time is given by a spatially-flat Robertson-Walker metric, which is the space observed by a low-energy observer on the brane world. In (1.3), $g_{s}$ is the string coupling, $M_{s}$ is the string state, and $M_{s} / g_{s}$ is the mass scale of the quantum gravitational foam fluctuations in the model. The metric (1.3) has precisely the form corresponding to a boosted frame (the D-particle's rest frame), with the boost occurring suddenly at time $t=t_{0}$, the time of the capture of the string state by the D-particle. On account of momentum conservation during the scattering [21], the D-particle recoil velocity $v_{i}$ is related to the momentum transfer $\Delta p_{i}$ of the string state, which in turn can be parametrized as a fraction of the incident momentum[22]

$$
v_{i}=g_{s} \frac{\Delta p_{i}}{M_{s}} \equiv r_{i} p_{i} \equiv g_{s} \frac{\xi_{i} p_{i}}{M_{s}}, \quad \text { no sum over } \mathrm{i}=1,2,3, r_{i}<1,
$$


where $p_{i}$ is a co-variant co-moving momentum of the DM particle in the (distorted) Robertson-Walker background. In our foam model we assume that the population of D-particles, is approximately uniform and relatively dense over a given epoch of the Universe. In such a case one may average (1.3) using appropriate distribution functions. Denoting such an average by $\ll \cdots \gg$, and assuming stochastic Gaussian distributions,

$$
\ll r_{i} \gg=0, \quad \ll r_{i} r_{j} \gg=\sigma_{i}^{2} \delta_{i j}, \quad \text { no sum over } \mathrm{i}=1,2,3 \text {. }
$$

The variances $\sigma_{i}^{2}$ need not be independent of $i$; however to keep in line with the observed isotropy of the Universe at large scales, such potential anisotropies (due to an anisotropy of the population of D-particles in the bulk [8, 9]) would have to be small.

Upon considering such foam populations, where the time scale between D-particle captures and emissions is much shorter than the inverse mass of the DM, a coarse-grained description is applicable; the propagation of a DM distribution over such a background is described on average by metrics $\ll d s^{2} \gg$ in which to a good approximation $t_{0} \sim t$. Consequently we write the distorted metric in the following form:

$$
g_{\mu \nu}=\left(\begin{array}{cccc}
-1 & a^{2}(t) r_{1} p_{1} & a(t)^{2} r_{2} p_{2} & a^{2}(t) r_{3} p_{3} \\
a^{2}(t) r_{1} p_{1} & a^{2}(t) & 0 & 0 \\
a^{2}(t) r_{2} p_{2} & 0 & a^{2}(t) & 0 \\
a^{2}(t) r_{3} p_{3} & 0 & 0 & a^{2}(t)
\end{array}\right) .
$$

The averaging over foam populations is performed using (1.5); hence, when considering the evolution of DM densities in such backgrounds, terms with an odd number of $r_{i}$ 's will be ignored. This should be understood in our subsequent discussion. The momentum dependence of the metric (1.6) implies a modification of the form of the geodesic equation for $\frac{d^{2} x^{\mu}}{d \tau^{2}}$ together with the modification of the Christoffel symbols. Keeping terms up to order $r^{2}$ and dropping everywhere cross terms of the form $r_{i} r_{j}$ for $i \neq j$ (these terms will in any case vanish when one takes the average), it is straightforward to show that the pertinent geodesic equation for $\mu=i(=1,2,3)$ reads:

$$
\begin{aligned}
& \frac{d^{2} x^{i}}{d \tau^{2}}=-\frac{2}{m^{2}} H p^{i} p^{0}-\frac{2}{m^{2}} a^{2}(t) H r_{i} p^{i}\left(p^{0}\right)^{2}+\frac{8}{m^{2}} a^{6}(t) H r_{i}^{2}\left(p^{i}\right)^{3} p^{0} \\
& +\frac{2}{m^{2}} a^{4}(t) H r_{i} p^{i} \sum_{j}\left(p^{j}\right)^{2}+\frac{4}{m^{2}} a^{4}(t) H r_{i}^{2} p^{i}\left(p^{0}\right)^{3}-\frac{4}{m^{2}} a^{6}(t) H r_{i}^{2} p^{i} p^{0} \sum_{j}\left(p^{j}\right)^{2}
\end{aligned}
$$

where the Einstein summation convention is not applied.

To discuss DM relics we need to solve the relevant Boltzmann equation that describes the evolution of the phasespace density function of the DM species in $t$. The distribution function $f$ of a particle species is specified by phase space variables of the system; it is thus natural to define a local space-time momentum in an expanding universe [23]:

$$
\bar{p}^{i} \equiv a(t) p^{i}, \quad i=1,2,3
$$

In terms of these scaled momenta, the energy-momentum dispersion relation for a DM particle of mass $m$ in our (spatially flat Robertson-Walker space-time background), assumes an effectively "Minkowski-space-time" form, $p^{\mu} p^{\nu} g_{\mu \nu}=-E^{2}+a^{2}(t) p^{i} p^{j} \delta_{i j}=-E^{2}+\bar{p}^{i} \bar{p}^{j} \delta_{i j}=-m^{2}$. Thus, it is essential to define the phase-space densities as functions of the coordinates $x^{\mu}=\left(x^{i}, t\right)$ and the local momenta $\bar{p}^{i}, f\left(x^{\mu}, \bar{p}^{i}\right)$. This choice of variables is particularly important in keeping the correct scaling properties of the DM density with the scale factor. In the usual isotropic Robertson Walker background, the momenta are assumed on-shell, and so the phase-space density depends on $|\vec{p}|$ (i.e. $E)$ rather than $\overrightarrow{\bar{p}}$. However, in our case, the small anisotropies that characterize the foam fluctuations are taken into account by assuming a dependence on the individual components $\bar{p}_{i}$.

The Liouville operator acting on $f\left(x^{\mu}, \bar{p}^{i}\right)$ takes the form:

$$
\hat{L}[f]=p^{\mu} \frac{\partial f}{\partial x^{\mu}}+m \sum_{i} \frac{\partial f}{\partial \bar{p}^{i}} \frac{d \bar{p}^{i}}{d \tau} .
$$

We apply the isotropy condition, $\frac{\partial f}{\partial x^{i}}=0, i=1,2,3$, and note that $\frac{d \bar{p}^{i}}{d \tau}=a(t) \frac{d p^{i}}{d \tau}+\dot{a}(t) \frac{d t}{d \tau} p^{i}=a(t) \frac{d p^{i}}{d \tau}+\dot{a}(t) \frac{\bar{p}^{0}}{m} p^{i}$; the following expression for the Liouville evolution operator is obtained:

$$
\begin{aligned}
& \frac{\hat{L}[f]}{p^{0}}=\frac{\partial f}{\partial t}-H \sum_{i} \bar{p}^{i} \frac{\partial f}{\partial \bar{p}^{i}}-2 H a^{2}(t) p^{0} \sum_{i} r_{i} \bar{p}^{i} \frac{\partial f}{\partial \bar{p}^{i}}+8 H a^{4}(t) \sum_{i} r_{i}^{2}\left(\bar{p}^{i}\right)^{3} \frac{\partial f}{\partial \bar{p}^{i}} \\
& +\frac{2}{p^{0}} H a^{2}(t) \sum_{j}\left(\bar{p}^{j}\right)^{2} \sum_{i} r_{i} \bar{p}^{i} \frac{\partial f}{\partial \bar{p}^{i}}+4 H a^{4}(t)\left(p^{0}\right)^{2} \sum_{i} r_{i}^{2} \bar{p}^{i} \frac{\partial f}{\partial \bar{p}^{i}}-4 a^{4}(t) H \sum_{j}\left(\bar{p}^{j}\right)^{2} \sum_{i} r_{i}^{2} \bar{p}^{i} \frac{\partial f}{\partial \bar{p}^{i}}
\end{aligned}
$$


The mass shell condition gives the energy dispersion relation

$$
p^{0}=a^{2}(t) \sum_{i}\left(\bar{p}^{i}\right)^{2} r_{i}+\sqrt{\sum_{i}\left(\bar{p}^{i}\right)^{2}+m^{2}}\left[1+\frac{a^{4}(t)\left(\sum_{i}\left(\bar{p}^{i}\right)^{2} r_{i}\right)^{2}}{\sum_{i}\left(\bar{p}^{i}\right)^{2}+m^{2}}\right]^{\frac{1}{2}}
$$

Further analysis involves the approximation of heavy DM, which we consider in this work, as the phenomenologically dominant species are the heavy ones that in general are expected to cause the most significant distortions in the space time background: $m^{2} \gg \sum_{i} \bar{p}^{i 2}$. In this case expanding (1.11) up to second order and averaging over the ensembles ${ }^{1}$, for the random variables $r_{i}$, we obtain for the Boltzmann equation (now with the binary collision term taken into account):

$$
\begin{aligned}
& \frac{\partial f}{\partial t}-H \sum_{i} \bar{p}^{i} \frac{\partial f}{\partial \bar{p}^{i}}+6 H a^{4}(t) \sum_{i} \sigma_{i}^{2}\left(\bar{p}^{i}\right)^{3} \frac{\partial f}{\partial \bar{p}^{i}}-\frac{2}{m^{2}} H a^{4}(t) \sum_{j}\left(\bar{p}^{j}\right)^{2} \sum_{i} \sigma_{i}^{2}\left(\bar{p}^{i}\right)^{3} \frac{\partial f}{\partial \bar{p}^{i}}+4 H m^{2} a^{4}(t) \sum_{i} \sigma_{i}^{2} \bar{p}^{i} \frac{\partial f}{\partial \bar{p}^{i}} \\
& -4 H a^{4}(t) \sum_{j}\left(\bar{p}^{j}\right)^{2} \sum_{i} \sigma_{i}^{2} \bar{p}^{i} \frac{\partial f}{\partial \bar{p}^{i}}=\frac{C[f]}{p^{0}}
\end{aligned}
$$

To make the connection with 1.1 the number density $n(t)$ is defined as

$$
n(t) \equiv \frac{g}{(2 \pi)^{3}} \int d^{3} \bar{p} f\left(t, \bar{p}^{i}\right)
$$

where $d^{3} \bar{p} \equiv d \bar{p}^{1} d \bar{p}^{2} d \bar{p}^{3}$. Following [24] an average temperature $T$ is defined through the equation

$$
\frac{g}{(2 \pi)^{3}} \int d^{3} \bar{p}\left(\bar{p}^{i}\right)^{2} f \equiv T m n .
$$

In the case of superheavy dark matter, the fourth and sixth terms in (1.12) are small compared to the others (and so will be neglected). Integrating (1.12) with respect to $d^{3} \bar{p}$, yields:

$$
\frac{d n}{d t}+3 H n=\Gamma(t) n+\frac{g}{(2 \pi)^{3}} \int d^{3} \bar{p} \frac{C[f]}{E}
$$

where we have incorporated all extra corrective terms in a time-dependent source term given by:

$$
\Gamma(t)=H a^{4}(t)\left(\sum_{i} \sigma_{i}^{2}\right)\left[18 T m+4 m^{2}\right],
$$

and

$$
\mathcal{C}[n]=\frac{g}{(2 \pi)^{3}} \int d^{3} \bar{p} \frac{C[f]}{E} .
$$

The reader should bear in mind that in our analysis we have kept only leading order terms in the fluctuations. Moreover, the correct scaling of $n$ with the Hubble parameter, embodied in the term $3 H n$ on the left-hand-side of (1.15), has been obtained because the local momentum $\bar{p}^{j}$ has been used in the argument of the phase-space distribution function $f\left(x^{i}, t, \bar{p}^{j}\right)[23]$, as mentioned earlier.

Hence the rôle of the stochastically fluctuating induced Finsler metrics in the D-particle foam model is simply to give rise to particle-production source terms in the Boltzmann equations, linear in the DM density. The presence of these terms (or equivalently the metric distortions) will affect quantities such as the thermal relic abundances (which will be distinguished with a prime). Although formally the situation is similar to that of time-dependent dilaton and non-critical string sources encountered in the super critical string (SSC) cosmology model of [17], our modification

\footnotetext{
1 The reason why we can do this at the equation level is because the time scale of D-particle scatterings is assumed to be much shorter than the Hubble time $H^{-1}$.
} 
$\Omega_{\chi}^{\prime}$ of the thermal relic abundance of a single heavy DM species $\chi$ will carry extra terms as compared to [17]. The process we follow is presented analytically in [25]. In a standard notation, the result is:

$$
\frac{\Omega_{\chi}^{\prime} h_{0}^{2}}{\left(\Omega_{\chi} h_{0}^{2}\right)_{\text {no source }}}=\left(1+\int_{x_{f}}^{x_{0}} \frac{\Gamma(x)}{H x} d x-\frac{1}{J} \int_{x_{f}}^{x_{0}} J(y)\left(\int_{x_{f}}^{y} \frac{\Gamma(x)}{H x} d x\right) d y\right)\left\{\left(\frac{g_{\text {eff }}^{\prime}}{g_{\text {eff }}}\right)_{x=x_{f}}\right\}^{\frac{1}{2}}
$$

where $J \equiv \int_{x_{0}}^{x_{f}}\langle v \tilde{\sigma}\rangle^{\prime} d x, J(x) \equiv \frac{\langle v \tilde{\sigma}\rangle^{\prime}}{x^{2}}$ and $x \equiv m_{\chi} / T$, following standard usage; $x_{0}$ denotes the current value of $x$ corresponding to the $\mathrm{CMB}$ temperature $T_{\mathrm{CMB}} \simeq 2.7^{0} \mathrm{~K}$, and $x_{f}$ denotes the (D-foam-modified) freeze-out temperature, estimated by using the freeze-out criterion $Y\left(x_{f}\right)-Y_{\text {eq }}\left(x_{f}\right) \approx c_{0} Y_{\text {eq }}\left(x_{f}\right)$, where $Y(x)=\frac{n(x)}{s}$, with $s$ an entropy density (satisfying $\frac{d}{d t}\left(s a^{3}\right)=0$ ) and the suffix "eq" denotes the equilibrium expressions (in the presence of the deformed metric) whose analytic form is given in [25]. The quantity $c_{0}$ is a phenomenological constant of order $\mathcal{O}(1)$, which can be determined by appropriate numerical fits. An analytic expression for $x_{f}$ is difficult to obtain in our case [25]; however, for weak foam effects, approximate expressions can be found by an appropriate expansion ${ }^{2}$. The quantity $\left(\Omega_{\chi} h_{0}^{2}\right)_{\text {no source }} \equiv \frac{1.066 \times 10^{9} \mathrm{GeV}^{-1}}{M_{P}} \sqrt{g_{\text {eff }}} J$ denotes the relic density in the absence of a source term $\Gamma=0$ and $J \equiv \int_{x_{0}}^{x_{f}}\langle v \tilde{\sigma}\rangle^{\prime} d x$. However, the reader should bear in mind that what we denoted as $\left(\Omega_{\chi} h_{0}^{2}\right)_{\text {no source }}$ is not quite the standard expression within the FRW Cosmology, since $\langle v \tilde{\sigma}\rangle^{\prime}$ carries implicit information about the D-particles effects on the total cross section (denoted by a prime) and also the freeze-out point that appears as the upper end of integration is shifted as described above; $M_{P}$ is the Planck mass, which is related to the D-particle mass scale $M_{s} / g_{s}$ via

$$
M_{P}^{2}=\frac{M_{s}^{2+\delta}}{g_{s}^{2+\delta}} V_{c}^{(\delta)}
$$

with $V_{c}^{(\delta)}$ the volume of the compactified $\delta$-extra dimensions of the 3-brane world, which depends on the specific model under consideration; the $M_{P}^{2}\left(=1 / G_{N}\right)$ enters the formalism through the effective four-dimensional EinsteinFriedmann equation $H^{2}=\frac{8 \pi G_{N}}{3}(\rho+\Delta \rho)$. Here, $\rho$ denotes the total (critical) radiation and matter energy density, including DM, and $\Delta \rho$ symbolizes collectively all the stochastic effects of the D-foam on the standard Einstein's equations. Regarding the latter, we note a small but important difference compared with the case of the timedependent dilaton model of [17]. The stochastic fluctuations of the space-time due to the recoil-velocity fluctuations of the D-particle defects in the D-foam, do contribute to thermalization of the Universe, indirectly, as a result of their coupling with the photon or electrically-neutral matter excitations (such as DM) via the distorted Finsler metric backgrounds ${ }^{3}$.

In order to calculate the explicit form of the factor $\left(\frac{g_{e f f}^{\prime}}{g_{e f f}}\right)_{x=x_{f}}$ that contributes to the D-foam corrections to dark matter relic abundances, one should first derive the equilibrium expressions for relativistic fermions and bosons in the

${ }^{2}$ For instance, it can be shown that the freeze-out criterion can be written as [25]: $\int_{x_{\text {in }}}^{x_{f}} \exp \left(\int_{x_{f}}^{y} \frac{x^{\prime} \Gamma\left(x^{\prime}\right)}{H_{m}} d x^{\prime}\right) \frac{\tilde{\sigma}(y) u_{0}}{H_{m} y^{2}} d y=\left(c_{0}+\right.$ $1)^{-1} u_{0} x_{f}^{-3} n_{\mathrm{eq}}^{-1}\left(x_{f}\right)$ where $\Gamma(x)$ is the source [1.16), the total cross section $\tilde{\sigma}(x) \sim \tilde{\sigma}_{0} x^{-j}, j=0(1)$ for $s(p)$-wave annihilators [19], $u_{0} \equiv \frac{2 \pi^{2}}{45} h^{\prime} m^{3}$, where $h^{\prime}$ denotes the entropy degrees of freedom, and we used $H=H_{m} x^{-2}, H_{m}=1.67 g_{\text {eff }}^{1 / 2} \frac{m^{2}}{M_{P}}$, with $M_{P}$ the Planck mass; $x_{\text {in }}$ corresponds to some initial value of the (inverse) temperature, say at the end of the inflationary era. The equilibrium number density $n_{\mathrm{eq}}(x)$ receives corrections from the foam [25]. For weak-foam situations, corresponding to large values of $M_{s} / g_{s}$ compared to the DM mass $m$, one may expand the exponential to first order in $\Gamma(x)$, taking into account that the scale factor $a(t) \sim 1 / T(c . f$. below), and solve iteratively for $x_{f}$. On assuming that the fitting constant $c_{0}$ can be chosen in such a way that the foam corrections on the right-hand-side of the above freeze-out criterion equation are subleading compared to their counterparts on the left-hand-side, one finds self-consistently [25] that the freeze-out point $x_{f}$ slightly increases relative to its foam-free-case value, $x_{f}^{(0)}$ (which for neutralino dark matter is of order $20[26]): x_{f}^{(j)} \simeq x_{f}^{(0),(j)}+\frac{6}{j+1}\left(g_{s} \frac{m}{M_{s}}\right)^{2}\left(\frac{x_{f}^{(0)}}{x_{0}}\right)^{4}\left(\frac{x_{f}^{(0)}}{x_{\mathrm{in}}}\right)^{j+1}\left(\sum_{i=1}^{3} \Delta_{i}^{2}\right)$, where $j=0(1)$ for $s-\quad(p-)$ annihilators, and $\Delta_{i}^{2}$ is a dimensionless foam-fluctuation variance (c.f. (1.4)). Hence, the foam leads to a relative decrease of the freeze-out temperature. However, taking into account that for neutralino masses in the range of $\mathcal{O}\left(10^{2}\right) \mathrm{GeV}[26], x_{\text {in }} \sim 10^{-12}$ (since one can reasonably place the end of inflation at temperatures of order $10^{14} \mathrm{GeV}$ ), and today's CMB value $x_{0} \sim 10^{14}$, one observes that the shift in the freeze-out temperature is negligible, for $\Delta_{i}^{2} \leq 1$, even for low string scales of order TeV.

${ }^{3}$ In our D-foam models [8-10] charged matter cannot couple dominantly to the D-particles due to electric charge flux conservation. 
presence of the D-foam. To this end, we use the basic formula [25]:

$$
\rho=\frac{g}{(2 \pi)^{3}} \int \ll n \omega_{r} \gg d^{3} \bar{p}
$$

where $\omega_{r}$ is the equivalent of (1.11) with $a(t)=1$ and the notation $\ll \ldots \gg$ denotes the average with respect to the statistical parameters $r_{i}$ of the foam i.e. :

$$
\ll n \omega_{r} \gg \equiv \prod_{j} \frac{1}{\sigma_{j} \sqrt{2 \pi}} \int_{-\infty}^{\infty} d r_{j}<n \omega>_{r} \exp \left(-\frac{r_{j}^{2}}{2 \sigma_{j}^{2}}\right)
$$

and (for any member of the ensemble of possible foams) we have the canonical energy distribution

$$
<n \omega>_{r}=\frac{\omega_{r}}{\exp \left(\beta\left(\omega_{r}-\mu\right)\right)+\varpi}
$$

where $\varpi=+1(-1)$ applies to fermions (bosons) and $\beta=\frac{1}{k_{B} T}$.

In this way we then obtain the expression [25]:

$$
\begin{aligned}
& \rho=\frac{g}{\pi^{2}}\left(\beta^{-4} \frac{1}{2} f_{3}(1, \beta \mu, \varpi)+\bar{\sigma}^{2} \beta^{-6}\left(\frac{1}{12} f_{5}(1, \beta \mu, \varpi)-\frac{1}{5} f_{6}(1, \beta \mu, \varpi)+\frac{1}{5} \xi f_{6}(2, \beta \mu, \varpi)-\frac{1}{12} f_{6}(1, \beta \mu, \varpi)\right.\right. \\
& \left.\left.+\frac{1}{12} \xi f_{6}(2, \beta \mu, \varpi)-\frac{1}{5} f_{7}(1, \beta \mu, \varpi)+\frac{1}{5} \xi f_{7}(2, \beta \mu, \varpi)+\frac{2}{5} f_{7}(1, \beta \mu, \varpi)-\frac{4}{5} \xi f_{7}(2, \beta \mu, \varpi)+\frac{2}{5} \xi^{2} f_{7}(3, \beta \mu, \varpi)\right)(1) .21\right)
\end{aligned}
$$

where $g$ denotes the spin degrees of freedom of either the fermions or the bosons under consideration and we have set $: \bar{\sigma}^{2} \equiv \sigma_{1}^{2}+\sigma_{2}^{2}+\sigma_{3}^{2}$. The functions $f_{j}$ appearing in (1.21) are defined through integrals of the general form:

$$
f_{j}(l ; \mu, \xi) \equiv \int_{0}^{\infty} d k \frac{k^{j}}{\left(e^{k-\mu}+\xi\right)^{l}}
$$

that can be related to $\zeta$ and Dirichlet $\eta$ functions. We also note here that since we are interested in relativistic species (contributing to $s$ ) we can set the chemical potential to $0\left(\mu \ll \omega_{r}\right)$ in (1.21) $\cdot g_{\text {eff }}^{\prime}$, the modified effective number of degrees of freedom, is determined from $\rho_{t o t}$, the total relativistic energy density, since $\rho_{t o t}=\frac{\pi^{2}}{30} g_{\text {eff }}^{\prime} T^{4}$; one finds that:

$$
g_{e f f}^{\prime}=g_{e f f}+\frac{30}{\pi^{2}} \bar{\sigma}^{2}\left(\frac{2 \pi^{4}}{189} \sum_{i} g_{i, b}\left(\frac{T_{i, b}}{T}\right)^{4} T_{i, b}^{2}+\frac{793.92}{\pi^{2}} \sum_{j} g_{j, f}\left(\frac{T_{j, f}}{T}\right)^{4} T_{j, f}^{2}\right)
$$

where $g_{\text {eff }}$ stands for the standard effective number of degrees of freedom[19] in the absence of D-foam corrections:

$$
g_{\text {eff }}=\sum_{i} g_{i, b}\left(\frac{T_{i, b}}{T}\right)^{4}+\frac{7}{8} \sum_{j} g_{j, f}\left(\frac{T_{j, f}}{T}\right)^{4}
$$

The numerical factors appearing in (1.23) are the result of calculating the values of the integrals (1.22). A detailed analysis of all these will appear in [25]. At temperatures of interest (since a freeze-out temperature is of the order of a few $\mathrm{GeV}$ for typical dark matter candidates with masses in the range $m \approx 1 \mathrm{GeV}-10^{4} \mathrm{GeV}$ [26]) all the species in the standard model behave as relativistic matter. A counting of degrees of freedom, then, yields: $g_{f}=g_{\text {quarks }}+g_{\text {leptons }}=$ $6 \times(2 \times 2 \times 3)+3 \times(2 \times 2)+3 \times 2=90$ and $g_{b}=g_{\text {gluons }}+g_{E W}+g_{\text {photon }}+g_{\text {Higgs }}=8 \times 2+3 \times 3+2+1=28$. Substituting these values in equations (1.23) and (1.24) and using the approximations $\frac{T_{i, b}}{T} \approx \frac{T_{j, f}}{T} \approx 1$ we find: $g_{\text {eff }}=106.75$ and $g_{\text {eff }}^{\prime}=106.75+22138 \bar{\sigma}^{2} T^{2}$. These considerations imply that the D-foam corrections to the effective degrees of freedom will be of order $\left(\frac{g_{e f f}^{\prime}}{g_{e f f}}\right)_{x_{f}} \approx 1+207.38 \bar{\sigma}^{2} T_{f}^{2}$. The dimensionful variances $\sigma_{i}^{2}$ are naturally suppressed by the (square) of the heavy D-particle mass scale (c.f. (1.4), with $\ll \xi_{i}^{2} \gg \equiv \Delta_{i}^{2}, i=1,2,3$ a dimensionless variance, that could be naturally up to $\mathrm{O}(1))$. Thus, we obtain:

$$
\left(\frac{g_{\text {eff }}^{\prime}}{g_{\text {eff }}}\right)_{x_{f}} \sim 1+207.38 g_{s}^{2} \frac{m^{2}}{M_{s}^{2}} x_{f}^{-2}\left(\sum_{i=1}^{3} \Delta_{i}^{2}\right) .
$$


To determine the final corrections to the relic abundances we may expand the relevant expressions up to order $\sigma_{i}^{2}$. Expressing the result in terms of $\Delta_{i}^{2}$, we finally obtain ${ }^{4}$ :

$$
\frac{\Omega_{\chi}^{\prime} h_{0}^{2}}{\left(\Omega_{\chi} h_{0}^{2}\right)_{\text {no source }}} \simeq\left[1+207.38 g_{s}^{2} \frac{m^{2}}{M_{s}^{2}} x_{f}^{-2}\left(\sum_{i=1}^{3} \Delta_{i}^{2}\right)\right]^{1 / 2}\left[1+g_{s}^{2} \frac{m^{2}}{M_{s}^{2}}\left(\sum_{i=1}^{3} \Delta_{i}^{2}\right)\left(1+6 x_{0}^{-1}\right)\right]
$$

where clearly the dominant correction terms are of order $g_{s}^{2} \frac{m^{2}}{M_{s}^{2}}\left(\sum_{i=1}^{3} \Delta_{i}^{2}\right)>0$. As noted in 1.18 it is possible to choose

From the positive signature of the foam-induced corrections we observe that the relic abundances are larger than the corresponding ones evaluated within a standard cosmological model. In this sense, the space-time foam background will leave less freedom for supersymmetry at colliders [17]. This effect is opposite to those induced by a time-dependent dilaton in supercritical string cosmologies [17]. In the latter case, the negative signature of the pertinent corrections, implied that there was less DM available today as compared to the standard cosmology calculations; this could then lead to much heavier supersymmetric partners produced at colliders, such as the LHC, with falsifiable signatures [18]. However, the corrections due to D-foam are in general small, as expected, and they can only be significant for low string scale models and heavy DM candidates. Indeed, the string scale $M_{s}$ is a free parameter in the modern version of string theory ( $\mathrm{cf}(1.18)$ ). For traditionally high string scales $\left(M_{s} \geq O\left(10^{16}\right) \mathrm{GeV}\right)$, in order for the D-foam effects to be significant one needs superheavy DM, with masses higher than $M_{s} / g_{s}$. However, the effects of such a superheavy DM will be eroded by inflation; moreover superheavy DM would not be produced significantly during a reheating phase of the Universe after its exit from the inflationary period. For intermediate string scales [27], where the quantity $M_{s} / g_{s}$ could be of order $10^{11} \mathrm{GeV}$ (which is the order of the GZK cutoff of ultra-high energy cosmic rays), there could be significant modifications in the relic abundances of superheavy DM particles with masses of this order. Such super-heavy DM particles can be produced during reheating [28], but in view of our scenario above, their relic abundance will be modified from the standard cosmology result. The presence of super-heavy DM, with increased relic abundances, might provide an explanation for the production of at least part of the spectrum of the ultra high energy cosmic rays, with energies of order $10^{20} \mathrm{eV}$. Hence, the effects of D-foam on such scenarios are worthy of investigating further, especially in view of the fact that the density of D-particles might be significantly higher at earlier eras of the Universe, leading to stronger stochastic effects $O\left(\Delta_{i}^{2}\right)$.

For low string scales, of order a few $\mathrm{TeV}$, the effects of the D-foam on thermal relic densities would be more significant. In fact, depending on the type of DM considered the effect could be constrained or falsified already by the WMAP five year data, since the induced increase of thermal relic abundance leaves less room for supersymmetry in the relevant parameter space. In certain cases, it may exceed the allowed region set by WMAP. The situation may thus lead to modifications of supersymmetry searches at colliders, especially in the context of neutralino DM models with the neutralino being Higgsino- or Wino-like, with masses up to TeV. In such cases there may be other reasons for an increased relic abundance, for instance slepton co-annihilation [29], and in fact our effects of the foam are of comparable strength in some of these cases.

Acknowledgements The work of N.E.M. and S.S. is supported in part by the European Union through the Marie Curie Research and Training Network UniverseNet (MRTN-2006-035863) and that of A.V. by a King's College London

\footnotetext{
${ }^{4}$ We remark at this stage that (1.26) has been derived by calculating the integral in (1.17) under the assumption that the scale factor $a(t)$ evolves with temperature $T$ as $T \sim \frac{T_{0}}{a(t)}$, where $T_{0}$ denotes today's temperature. However, this is only approximately true, since particles' annihilation at different stages of the evolution of the universe slowed down the cooling due to heat deposition. Actually, the corrected cooling law, obtained by assuming that the entropy (which is dominated by the relativistic species contribution) remains constant despite the presence of the source, reads: $a(t) \approx\left(\frac{g_{\text {eff }, 0}}{g_{\text {eff }}(t)}\right)^{1 / 3} \frac{a_{0} T_{0}}{T}$. The function $g_{\text {eff }}(t)$ is known [19] and is such that $g_{\text {eff }, 0} \simeq 3.36$ (due to the fact that today only photons and the light neutrinos contribute), while at freeze-out $g_{\text {eff }}\left(t_{f}\right) \simeq 106.75$ within the framework of the Standard Model. In principle, one should calculate the integral in (1.17) numerically using this evolution of $g_{\text {eff }}(t(T))$. For our qualitative purposes in this work we shall ignore such corrections which, at any rate, do not affect significantly the order of magnitude of foam contributions to the relic abundances. To get an estimation of how big the difference is and assuming that the entropy will be conserved despite the presence of the source term (see also [17]) implies that $g_{\text {eff }} a^{3}(t) T^{3}$ will remain constant throughout the evolution of the universe (only relativistic species are connsidered to contribute to the entropy). Today only photons and neutrinos can contribute to the entropy yielding $g_{\text {eff }, 0}=3.36$. Therefore very roughly one would get a correction of the order of $\frac{(a(t) T)_{G e V}^{3}}{\left(a_{0} T_{0}\right)^{3}} \approx \frac{1}{30}$. This is fully taken into account in $[25]$
} 
Departmental Graduate Studentship.

[1] A. G. Riess et al. [Supernova Search Team Collaboration], Astron. J. 116, 1009 (1998); S. Perlmutter et al. [Supernova Cosmology Project Collaboration], Astrophys. J. 517, 565 (1999); A. G. Riess, Publ. Astron. Soc. Pac. 112, 1284 (2000); E. Linder and S. Perlmutter, Phys. World $20 N 12$ (2007) 24; M. Kowalski et al. [Supernova Cosmology Project Collaboration], Astrophys. J. 686, 749 (2008); W. M. Wood-Vasey et al. [ESSENCE Collaboration], Astrophys. J. 666, 694 (2007); A. G. Riess and M. Livio, Astrophys. J. 648, 884 (2006); A. G. Riess et al. [Supernova Search Team Collaboration], Astrophys. J. 607, 665 (2004).

[2] D. N. Spergel et al. [WMAP Collaboration], Astrophys. J. Suppl. 170, 377 (2007).

[3] D. J. Eisenstein et al. [SDSS Collaboration], Astrophys. J. 633, 560 (2005); H. J. Seo and D. J. Eisenstein, Astrophys. J. 598, 720 (2003); and references therein.

[4] L. Fu et al., Astronomy \& Astrophysics 479, 9 (2008); L. Guzzo et al., Nature 451, 541 (2008).

[5] J. A. Wheeler and K. Ford, Geons, Black Holes and Quantum Foam: A Life in Physics (Norton, New York, 1998).

[6] For a comprehensive review see: L. H. Ford, Int. J. Theor. Phys. 44, 1753 (2005) arXiv:gr-qc/0501081 and references therein.

[7] For reviews see: T. Jacobson, S. Liberati and D. Mattingly, Annals Phys. 321, 150 (2006); N. E. Mavromatos, J. Phys. Conf. Ser. 174, 012016 (2009) arXiv:0903.0318 [astro-ph.HE]], and references therein.

[8] J. R. Ellis, N. E. Mavromatos and D. V. Nanopoulos, Gen. Rel. Grav. 32, 127 (2000); Phys. Rev. D 61, 027503 (2000); Phys. Rev. D 62, 084019 (2000); J. R. Ellis, N. E. Mavromatos and M. Westmuckett, Phys. Rev. D 70, 044036 (2004); ibid. 71, 106006 (2005) .

[9] J. R. Ellis, N. E. Mavromatos and D. V. Nanopoulos, Phys. Lett. B 665, 412 (2008); arXiv:0912.3428 [astro-ph.CO].

[10] T. Li, N. E. Mavromatos, D. V. Nanopoulos and D. Xie, Phys. Lett. B 679, 407 (2009).

[11] See for instance: G. Shiu, L. -T. Wang, Phys. Rev. D69, 126007 (2004), and references therein.

[12] G. Amelino-Camelia, J. R. Ellis, N. E. Mavromatos, D. V. Nanopoulos and S. Sarkar, Nature 393, 763 (1998); J. R. Ellis, K. Farakos, N. E. Mavromatos, V. A. Mitsou and D. V. Nanopoulos, Astrophys. J. 535, 139 (2000); J. R. Ellis, N. E. Mavromatos, D. V. Nanopoulos, A. S. Sakharov and E. K. G. Sarkisyan, Astropart. Phys. 25, 402 (2006) [Astropart. Phys. 29, 158 (2008)].

[13] See, for instance: D. Bao, S. S. Chern and Z. Shen, An introduction to Finsler Geometry (Springer-Verlag (NY, 2000)). In the context of D-partidle foam, such Finsler-type metrics have been first derived in: J. R. Ellis, N. E. Mavromatos and D. V. Nanopoulos, Int. J. Mod. Phys. A 13, 1059 (1998); For a short recent review on this topic see: N. E. Mavromatos, PoS QG-PH, 027 (2007) [arXiv:0708.2250 [hep-th]] and references therein.

[14] N. Seiberg and E. Witten, JHEP 9909, 032 (1999); N. Seiberg, L. Susskind and N. Toumbas, JHEP 0006, 044 (2000).

[15] P. Nath, R. L. Arnowitt and A. H. Chamseddine, Nucl. Phys. B 227, 121 (1983); H. P. Nilles, Phys. Rept. 110, 1 (1984).

[16] J. R. Ellis, K. A. Olive, Y. Santoso and V. C. Spanos, Phys. Lett. B 565, 176 (2003) arXiv:hep-ph/0303043. A. B. Lahanas and D. V. Nanopoulos, Phys. Lett. B 568, 55 (2003) arXiv:hep-ph/0303130. For a review see: A. B. Lahanas, N. E. Mavromatos and D. V. Nanopoulos, Int. J. Mod. Phys. D 12, 1529 (2003).

[17] A. B. Lahanas, N. E. Mavromatos and D. V. Nanopoulos, PMC Phys. A 1 (2007) 2; Phys. Lett. B 649, 83 (2007).

[18] B. Dutta, A. Gurrola, T. Kamon, A. Krislock, A. B. Lahanas, N. E. Mavromatos and D. V. Nanopoulos, Phys. Rev. D 79, 055002 (2009).

[19] E. W. Kolb and M. S. Turner, Front. Phys. 691 (1990).

[20] E. Gravanis and N. E. Mavromatos, JHEP 0206, 019 (2002);

[21] I. I. Kogan, N. E. Mavromatos and J. F. Wheater, Phys. Lett. B 387, 483 (1996); I. I. Kogan and N. E. Mavromatos, Phys. Lett. B 375, 111 (1996); N. E. Mavromatos and R. J. Szabo, Phys. Rev. D 59, 104018 (1999); N. E. Mavromatos, Logarithmic conformal field theories and strings in changing backgrounds, in Shifman, M. (ed.) et al.: From fields to strings, I. Kogan memorial Volume 2, 1257-1364. (World Sci. 2005), and references therein. arXiv:hep-th/0407026.

[22] N.E. Mavromatos and Sarben Sarkar, Phys. Rev. D 72, 065016 (2005) arXiv:hep-th/0506242.

[23] J. Bernstein, Kinetic theory in an Expanding Universe (Cambridge Univ. Press, 1988).

[24] M.Krook and T.T. Wu, The Physics of Fluids, 691589 (1977).

[25] N. E. Mavromatos, Sarben Sarkar and A. Vergou, in preparation.

[26] A. B. Lahanas, D. V. Nanopoulos and V. C. Spanos, Phys. Rev. D 62 (2000) 023515 arXiv:hep-ph/9909497.

[27] I. Antoniadis and B. Pioline, Nucl. Phys. B 550, 41 (1999).

[28] D. J. H. Chung, E. W. Kolb and A. Riotto, Phys. Rev. D 60, 063504 (1999).

[29] S. Profumo and A. Provenza, JCAP 0612, 019 (2006). 